\title{
Simulation of Three-Dimensional Internal Flows by the Random Vortex and Boundary Element Methods
}

\author{
Adrin Gharakhani and Ahmed F. Ghoniem \\ Department of Mechanical Engineering \\ Massachusetts Institute of Technology \\ Cambridge, MA 02139, USA
}

\begin{abstract}
A vortex boundary element method is developed for the grid-free simulation of time-dependent, incompressible, viscous flow in three-dimensional configurations. The numerical scheme is based on a combination of the Lagrangian vortex method to capture the convection and stretch of the vortical field, the random walk method to describe the diffusion process, and the boundary element method to impose the normal flux boundary condition on the boundary surfaces. The no-slip boundary condition is satisfied by an extended vortex tile generation mechanism. A new boundary condition is devised to impose the fully developed flow properties at the exit plane. The formulation of the numerical scheme is presented, followed by a parametric study of the accuracy of the method using the model problem of flow in a duct with square cross-section at $R e=100$. Additionally, results from an example of piston driven flow in a cylinder with square cross-section and an offset port at $R e=350$ (based on the piston side and maximum speed) are presented.
\end{abstract}

\section{Introduction}

Three-dimensional Lagrangian vortex methods were first attempted by simulating inviscid flow using a vortex filament approach [16]. In this case, a collection of filaments - each forming a closed vortex line and segmented along the vorticity vector - is used to approximate the initial vorticity field. Beale and Majda proposed extending the notion of a two dimensional vortex blob to a threedimensional blob with spherical core function and provided mathematical proof for the existence of solutions for short times [4]. However, the velocity gradients in the vorticity stretch term were obtained using a finite difference approximation in contradiction to the spirit of grid-free Lagrangian techniques. Anderson and Greengard suggested alleviating this problem by directly differentiating the regularized Biot-Savart integral for the velocity [1]. The latter approach and its variants have been used extensively in three-dimensional unbounded domains [15, 24].

Fishelov combined the method by Anderson and Greengard [1] with the three-dimensional extension of the vorticity generation scheme by Chorin [6,7] (for uniform tile distributions), and simulated turbulent flow over a flat plate [8]. Diffusion was approximated using the random walk method and the method of images was applied to impose the zero normal flux condition at the wall. Chorin performed a similar study using vortex filaments instead of blobs [7].

In this paper, a random vortex-boundary element method is used for the grid-free simulation of time-dependent incompressible viscous flow in three-dimensional configurations. (See [9] for details.) The random vortex method with a second order core function for the vorticity is applied to simulate the Navier-Stokes equations in an unbounded domain. The latter is modified by a 
potential flow such that the proper normal flux boundary condition is satisfied. The potential flow is obtained by the boundary element method, using piecewise linear or quadratic variation of the potential and the normal flux across the boundary elements. The no-slip boundary condition is satisfied by an extended tile generation mechanism that accounts for time-varying walls. In addition, a new approach for imposing a fully developed flow at the exit plane is proposed. The accuracy of the scheme is examined using the example of flow in a duct with square cross-section at $R e=100$. The versatility of the method is demonstrated using an example of flow inside a piston driven cylinder and an off-centered port (with square cross sections) at $R e=350$ based on the maximum speed of the piston and its width.

\section{Numerical formulation}

The equations of motion of an incompressible flow within a three-dimensional domain $D$, with boundary surfaces $\partial D$, are expressed in the vorticity transport form of the Navier-Stokes equations as follows:

$$
\begin{aligned}
\frac{\partial \omega}{\partial t}+\mathbf{u} \cdot \nabla \omega & =\omega \cdot \nabla \mathbf{u}+\frac{1}{R e} \nabla^{2} \omega, & & \mathbf{x} \in D \\
\nabla \cdot \mathbf{u} & =0, & & \mathbf{x} \in D \\
\mathbf{u}(\mathbf{x}, t) & =(\mathbf{u} \cdot \boldsymbol{\tau}, \mathbf{u} \cdot \boldsymbol{\rho}, \mathbf{u} \cdot \mathbf{n}), & & \mathbf{x} \in \partial D \\
\mathbf{u}(\mathbf{x}, t=0) & =\mathbf{u}_{o}, & & \mathbf{x} \in D
\end{aligned}
$$

where $\mathbf{x}=(x, y, z)$ is the position vector in the Cartesian coordinates normalized by a reference length $L ; \mathbf{u}(\mathbf{x}, t)=(u, v, w)$ is the velocity vector normalized by a characteristic speed $U ; t$ is the time normalized by $L / U$; and $\omega(\mathbf{x}, t)=\left(\omega_{x}, \omega_{y}, \omega_{z}\right)$ is the vorticity vector and is defined as the curl of the velocity vector, $\omega=\nabla \wedge \mathbf{u}$. Re $=U L / \nu$ is the Reynolds number and $\nu$ is the kinematic viscosity. At the boundary surfaces velocity is expressed in terms of the local orthogonal coordinate system $(\boldsymbol{\tau}, \boldsymbol{\rho}, \mathbf{n})$, where $\mathbf{n}=\left(n_{x}, n_{y}, n_{z}\right)$ is the unit outward normal, and $\boldsymbol{\tau}=\left(\tau_{x}, \tau_{y}, \tau_{z}\right)$ and $\boldsymbol{\rho}=\left(\rho_{x}, \rho_{y}, \rho_{z}\right)$ are the unit tangents to the boundary.

The transport of vorticity in the field is approximated by a domain decomposition technique, by which vorticity is discretized using a collection of vortex tiles in a thin region near the boundary and using a set of spherical elements in the domain interior - away from the boundary region. The two sub-domains are interlinked by converting the tiles into elements as they cross the interface between the two regions.

\subsection{The interior}

We begin the solution of Eq. 1 by discretizing the vorticity field using a collection of vortex elements, each centered at $\mathbf{x}_{j}$ with element volume $\Delta V_{j}$ and vorticity vector $\omega_{j}$ :

$$
\omega(\mathbf{x}, t)=\sum_{j=1}^{N_{V}} \tilde{\Gamma}_{j}(t) g_{\sigma}\left(\mathbf{x}-\mathbf{x}_{j}\right)
$$

where $\tilde{\Gamma}_{j}(t)=\omega_{j}(t) \Delta V_{j}$ is the volumetric vorticity and $g_{\sigma}(\mathbf{x})=\frac{1}{\sigma^{3}} g\left(\frac{|\mathbf{x}|}{\sigma}\right)$ is the vortex "blob" function with a spherical core of radius $\sigma$. The accuracy and convergence properties of the vortex blob method may be found in $[2,4,13,14,17]$. We used the second order core function $g(\mathbf{x})=$ $\frac{3}{4 \pi}\left[1-\tanh ^{2}\left(|\mathbf{x}|^{3}\right)\right]$ selected from a list of functions recommended by Beale and Majda [5]. 
The evolution of the vorticity field may be obtained to any order of accuracy in time by applying viscous splitting to the Navier-Stokes equations [3] in conjunction with vorticity distribution (2) as follows:

$$
\begin{aligned}
\chi_{i}^{*}\left(t_{k+1}\right) & =\chi_{i}\left(t_{k}\right)+\Delta t F\left[\mathbf{u}_{i}\left(\chi_{i}, t_{k}\right)\right], & \chi_{i}\left(t_{o}\right)=\chi_{i, o} \\
\tilde{\Gamma}_{i}\left(\chi_{i}, t_{k+1}\right) & =\tilde{\Gamma}_{i}\left(\chi_{i}, t_{k}\right)+\Delta t F\left[\tilde{\Gamma}_{i}\left(\chi_{i}, t_{k}\right) \cdot \nabla \mathbf{u}_{i}\left(\chi_{i}, t_{k}\right)\right], & \tilde{\Gamma}_{i}\left(\chi_{i}, t_{o}\right)=\tilde{\Gamma}_{i, o} \\
\chi_{i}\left(t_{k+1}\right) & =\chi_{i}^{*}\left(t_{k+1}\right)+\boldsymbol{\eta}_{i}(\Delta t), & i=1, \ldots, N_{V}, \quad k=0,1, \ldots
\end{aligned}
$$

where $\chi_{i}$ describes the trajectory of the $i$-th vortex element initially at $\chi_{i, o}$ and with vorticity $\tilde{\Gamma}_{i, o}$, $t_{o}$ is the time it is introduced into the domain, $\Delta t=t_{k+1}-t_{k}$ is the integration timestep, and $F[$.$] represents the integration scheme. We experimented with the second order modified Euler and$ fourth order Runge-Kutta methods and selected the former for its economy and acceptable accuracy. Equation (5) is the stochastic approximation for the diffusion equation and $\boldsymbol{\eta}_{i}=\left(\eta_{x}, \eta_{y}, \eta_{z}\right)_{i}$ are random variables in each of the three coordinate directions, selected independently from a Gaussian distribution with zero mean and variance equal to $2 \Delta t / R e$.

In Eqs. 3 and 4, the velocity and its gradients at the element centers are evaluated as the sum of a vortical field in free space and a potential flow such that continuity and the normal flux boundary condition are preserved. Given the vorticity distribution (2), the discrete regularized vortical velocity $\mathbf{u}_{\omega}$ and its gradients $\nabla \mathbf{u}_{\omega}$ are evaluated as follows:

$$
\begin{aligned}
\mathbf{u}_{\omega}\left(\mathbf{x}_{i}, t\right) & =\sum_{j=1}^{N_{V}} K_{\sigma}\left(\mathbf{x}_{i}-\mathbf{x}_{j}\right) \wedge \tilde{\Gamma}_{j}(t), & i=1, \ldots, N_{V} \\
\nabla \mathbf{u}_{\omega}\left(\mathbf{x}_{i}, t\right) & =\sum_{j=1}^{N_{V}} \nabla K_{\sigma}\left(\mathbf{x}_{i}-\mathbf{x}_{j}\right) \wedge \tilde{\Gamma}_{j}(t), & i=1, \ldots, N_{V}
\end{aligned}
$$

where

$$
\begin{gathered}
K_{\sigma}(\mathbf{x})=-\frac{\mathbf{x}}{4 \pi|\mathbf{x}|^{3}} f\left(\frac{|\mathbf{x}|}{\sigma}\right), \quad K_{\sigma}(0)=0, \quad \nabla K_{\sigma}(\mathbf{x})=\left(\frac{\nabla \mathbf{x}}{\mathbf{x}}-3 \frac{\nabla|\mathbf{x}|}{|\mathbf{x}|}\right) K_{\sigma}(\mathbf{x})-\mathbf{x} \frac{\nabla|\mathbf{x}|}{|\mathbf{x}|} g_{\sigma}(\mathbf{x}) \\
\nabla K_{\sigma}(0)=-\frac{\nabla \mathbf{x}}{4 \pi \sigma^{3}} \text { and } f(r)=4 \pi \int_{0}^{r} g\left(r^{\prime}\right) r^{\prime 2} d r^{\prime}=\tanh \left(r^{3}\right) .
\end{gathered}
$$

The potential component of the velocity $\mathbf{u}_{P}$ and its gradients $\nabla \mathbf{u}_{P}$ are obtained by solving a three-dimensional Neumann problem for the interior:

$$
\begin{array}{cc}
\nabla^{2} \Phi(\mathbf{x})=0, & \mathbf{x} \in D \\
q\left(\mathbf{x}_{o}\right)=\nabla \Phi\left(\mathbf{x}_{o}\right) \cdot \mathbf{n}=-\mathbf{u}_{P}\left(\mathbf{x}_{o}\right) \cdot \mathbf{n}=-\left(\mathbf{u}\left(\mathbf{x}_{o}\right)-\mathbf{u}_{\omega}\left(\mathbf{x}_{o}\right)\right) \cdot \mathbf{n}, & \mathbf{x}_{o} \in \partial D
\end{array}
$$

where $\Phi\left(\mathbf{x}_{o}\right)$ is the potential distribution, the gradient of which yields the potential velocity, and $q\left(\mathbf{x}_{o}\right)$ is its normal flux at the boundary. The details of the accurate solution of this problem by the direct boundary element method are given elsewhere [10]. Having obtained the potential distribution, the potential velocity and its gradients at the vortex element centers are evaluated using the following regularized formulations [10, 22, 23]:

$$
u_{P_{j}}\left(\mathbf{x}_{i}\right)=\sum_{k=1}^{M} \int_{\partial D_{k}}-G_{, p}\left(\mathbf{x}_{o}, \mathbf{x}_{i}\right)\left\{\varepsilon_{j p l}\left[\rho_{l}^{k} \Phi_{k, \tau}\left(\mathbf{x}_{o}\right)-\tau_{l}^{k} \Phi_{k, \rho}\left(\mathbf{x}_{o}\right)\right]-\delta_{j p} q_{k}\left(\mathbf{x}_{o}\right)\right\} d S_{k}\left(\mathbf{x}_{o}\right)
$$




$$
\begin{aligned}
& \frac{\partial u_{P_{j}}\left(\mathbf{x}_{i}\right)}{\partial x_{m}}= \sum_{k=1}^{M} \int_{\partial D_{k}}-G_{, p}\left(\mathbf{x}_{o}, \mathbf{x}_{i}\right)\left\{\varepsilon _ { j p l } \left[\rho_{l}^{k} \tau_{m}^{k} \Phi_{k, \tau \tau}\left(\mathbf{x}_{o}\right)-\tau_{l}^{k} \rho_{m}^{k} \Phi_{k, \rho \rho}\left(\mathbf{x}_{o}\right)+\right.\right. \\
&\left.\left(\rho_{l}^{k} \rho_{m}^{k}-\tau_{l}^{k} \tau_{m}^{k}\right) \Phi_{k, \tau \rho}\left(\mathbf{x}_{o}\right)+\left(\rho_{l}^{k} q_{k, \tau}\left(\mathbf{x}_{o}\right)-\tau_{l}^{k} q_{k, \rho}\left(\mathbf{x}_{o}\right)\right) n_{m}^{k}\right]+ \\
&\left.\delta_{j p}\left[q_{k, \tau}\left(\mathbf{x}_{o}\right) \tau_{m}^{k}+q_{k, \rho}\left(\mathbf{x}_{o}\right) \rho_{m}^{k}-\left(\Phi_{k, \tau \tau}\left(\mathbf{x}_{o}\right)+\Phi_{k, \rho \rho}\left(\mathbf{x}_{o}\right)\right) n_{m}^{k} q_{k}\left(\mathbf{x}_{o}\right)\right]\right\} d S_{k}\left(\mathbf{x}_{o}\right), \\
& i=1, \ldots, N_{V}, \quad(j, l, m, p)=1,2,3
\end{aligned}
$$

where $G\left(\mathbf{x}_{o}, \mathbf{x}_{i}\right)=\frac{1}{4 \pi\left|\mathbf{x}_{o}-\mathbf{x}_{i}\right|} ; j, l, m$ and $p$ indices indicate direction with respect to the global coordinate system and follow the Einstein rule; $(.)_{, r}$ represents differentiation with respect to $\mathbf{x}_{o}$ in the $r$-th direction, $\delta_{j p}$ is the Kronecker delta, and $\varepsilon_{j p l}$ is the antisymmetric permutation tensor. $M$ is the total number of rectangular boundary elements and $d S_{k}\left(\mathbf{x}_{o}\right)$ is the surface area of the $k$-th element. The first and second degree tangential derivatives of the potential and the normal flux are obtained by piecewise differentiation of the corresponding shape functions across each element.

\subsection{The boundary region}

In this section, all variables are defined with respect to the local coordinate system. $z$ is assigned normal to the wall and into the flow interior, with $z=0$ representing the wall surface. The vorticity distribution in the interior and the satisfaction of the normal flux boundary condition induce a tangential velocity $\left(u^{+}, v^{+}\right)$, which is generally different from the prescribed boundary condition $\left(u^{-}, v^{-}\right)$. This difference is the surface vorticity that must be generated to satisfy the no-slip boundary condition and it is discretized using a set of rectangular vortex tiles; each with sides $h_{x_{i}}^{t}$ and $h_{y_{i}}^{t}$, and with surface vorticity $\bar{\gamma}\left(x_{i}, y_{i}, 0, t\right)$ centered at $\left(x_{i}, y_{i}, 0\right)$. The tile vorticity is linked to the velocity jumps at the boundary element nodes as follows:

$$
\bar{\gamma}\left(x_{i}, y_{i}, 0, t\right)=\varepsilon_{k j 3} \sum_{m=1}^{M_{B}}\left(\overline{u_{k}^{+}-u_{k}^{-}}\right)_{m} \Lambda\left(x_{i}, x_{m}, h_{x_{i}}^{t}, h_{x_{m}}^{b}\right) \Lambda\left(y_{i}, y_{m}, h_{y_{i}}^{t}, h_{y_{m}}^{b}\right), \quad(j, k)=(1,2)
$$

where

$$
\Lambda\left(x_{i}, x_{m}, h_{x_{i}}^{t}, h_{x_{m}}^{b}\right)=\left[\frac{h_{x_{i}}^{t}+h_{x_{m}}^{b}}{2}-\max \left(\frac{h_{x_{i}}^{t}-h_{x_{m}}^{b}}{2}, \min \left(\left|x_{i}-x_{m}\right|, \frac{h_{x_{i}}^{t}+h_{x_{m}}^{b}}{2}\right)\right)\right] / h_{x_{i}}^{t}
$$

and $M_{B}$ is the number of boundary elements on the wall, each centered at $\left(x_{m}, y_{m}, 0\right)$ and with sides $h_{x_{m}}^{b}$ and $h_{y_{m}}^{b}$. The velocity overbars denote the area averaged values over the boundary elements. To maintain a finer discretization of the flow in the direction normal to the wall, each tile may be split into $N_{S, i}=\left(\left|\bar{\gamma}\left(x_{i}, y_{i}, 0, t\right)\right| / \gamma_{\max }+0.5\right)$ stacks of tiles with surface vorticity equal to $\tilde{\gamma}\left(x_{i}, y_{i}, 0, t\right)=\bar{\gamma}\left(x_{i}, y_{i}, 0, t\right) / N_{S, i}[8,11,12,19]$. $\gamma_{\max }$ is a predefined maximum surface vorticity the inverse of which is called the "Boundary Layer Resolution Parameter", BLRP, in this paper.

Once vorticity is generated at the wall, its evolution within the thin boundary region $D_{b}$ near the wall is assumed to be locally two dimensional and is approximated as follows $[7,8]$ :

$$
\begin{array}{ccc}
\frac{\partial \omega}{\partial t}+\mathbf{u} \cdot \nabla \omega & =\frac{1}{R e} \frac{\partial^{2} \omega}{\partial z^{2}}, & \mathbf{x} \in D_{b} \\
\nabla \cdot \mathbf{u}=0, & \mathbf{x} \in D_{b} \\
\mathbf{u}(x, y, z=0, t)=(0,0,0), \quad \mathbf{u}(x, y, z=b, t)=\left(U_{\infty}, V_{\infty}, 0\right) & \\
\mathbf{u}(\mathbf{x}, t=0)=\mathbf{u}_{o}, & \mathbf{x} \in D_{b}
\end{array}
$$


where $\omega(\mathbf{x}, t)=\left(\omega_{x}, \omega_{y}, \omega_{z}\right) \cong(-\partial v / \partial z, \partial u / \partial z, 0) .\left(U_{\infty}, V_{\infty}, 0\right)$ is the velocity at the edge of the boundary layer as seen by the wall and is obtained from the Navier-Stokes solution in the interior. $b=\mathrm{BLTC} \sqrt{2 \Delta t / R e}$ is a user specified boundary thickness and $1.0 \leq \mathrm{BLTC} \leq 3.0$, the "boundary layer thickness coefficient", is normally taken so that the tiles will jump into the flow interior in a few timesteps with relatively high probability $[8,18]$.

For a given tile distribution within $b$, the discretized $u$ and $v$ velocity components at the tile centers $\left(x_{i}, y_{i}, z_{i}\right)$ are derived by directly integrating the approximate definitions for $\omega_{y}$ and $\omega_{x}$, respectively, and applying the corresponding velocity boundary conditions:

$$
\begin{array}{r}
u_{j}\left(\mathbf{x}_{i}, t\right)=U_{j, \infty}\left(x_{i}, y_{i}, b, t\right)+\varepsilon_{k j 3}\left[\frac{1}{2} \tilde{\gamma}_{k}\left(\mathbf{x}_{i}, t\right)+\sum_{\substack{l=1 \\
l \neq i}}^{N_{T}} \tilde{\gamma}_{k}\left(\mathbf{x}_{i}, t\right) \phi_{l}\left(x_{i}, y_{i}\right) s\left(z_{l}-z_{i}\right)\right] \\
i=1, \ldots, N_{T}, \quad(j, k)=1,2
\end{array}
$$

where $\phi_{l}\left(x_{i}, y_{i}\right)=\Lambda\left(x_{i}, x_{l}, h_{x_{i}}^{t}, h_{x_{l}}^{t}\right) \Lambda\left(y_{i}, y_{l}, h_{y_{i}}^{t}, h_{y_{l}}^{t}\right), N_{T}$ is the total number of vortex tiles, $s$ is the step function, and $\left(U_{1, \infty}, U_{2, \infty}\right) \equiv\left(U_{\infty}, V_{\infty}\right)$. The $w$ component is obtained by satisfying continuity:

$$
w\left(\mathbf{x}_{i}, t\right)=-\frac{\left(I_{x}^{+}-I_{x}^{-}\right)}{h_{x_{i}}^{t}}-\frac{\left(I_{y}^{+}-I_{y}^{-}\right)}{h_{y_{i}}^{t}}, \quad i=1, \ldots, N_{T}
$$

where the divided difference rule is applied to approximate the derivatives and

$$
\begin{aligned}
& I_{x}^{ \pm}=U_{\infty}\left(x_{i} \pm h_{x_{i}}^{t} / 2, y_{i}, b, t\right) z_{i}-\sum_{l=1}^{N_{T}} \tilde{\gamma}_{y}\left(\mathbf{x}_{l}, t\right) \phi_{l}\left(x_{i} \pm h_{x_{i}}^{t} / 2, y_{i}\right) \min \left(z_{l}, z_{i}\right) \\
& I_{y}^{ \pm}=V_{\infty}\left(x_{i}, y_{i} \pm h_{y_{i}}^{t} / 2, b, t\right) z_{i}-\sum_{l=1}^{N_{T}} \tilde{\gamma}_{x}\left(\mathbf{x}_{l}, t\right) \phi_{l}\left(x_{i}, y_{i} \pm h_{y_{i}}^{t} / 2\right) \min \left(z_{l}, z_{i}\right)
\end{aligned}
$$

A first order Euler time integration is implemented to advance the tiles:

$$
\begin{array}{rlr}
\mathbf{x}_{i}^{*}\left(t_{k+1}\right) & =\mathbf{x}_{i}\left(t_{k}\right)+\mathbf{u}_{i}\left(\mathbf{x}_{i}, t_{k}\right) \Delta t & \mathbf{x}_{i}\left(t_{o}\right)=\left(x_{i}, y_{i}, 0, t_{o}\right) \\
z_{i}\left(t_{k+1}\right) & =\left|z_{i}^{*}\left(t_{k+1}\right)+\boldsymbol{\eta}_{i}\right| & i=1, \ldots, N_{T}, \quad k=0,1, \ldots
\end{array}
$$

where $\left(x_{i}, y_{i}, z_{i}\right)$ denotes the tile trajectory $\chi_{i}$ in the current context. Equation $7 \mathrm{~b}$ simulates diffusion normal to the wall and into the interior by reflecting tiles that jump below the wall back into the field [19].

The numerical algorithm proceeds as follows: During each timestep, vortex elements evolve according to Eqs. 3 through 5. The tiles convect concurrently with the vortex elements in the thin region near the wall according to Eq. 7a. New tiles are generated at the walls to satisfy the no-slip boundary condition. Finally, all tiles (old and new) diffuse toward the flow interior according to Eq. 7b, signifying the end of the time-step. If a tile jumps into the flow interior it is converted into a vortex blob with volumetric vorticity $\tilde{\Gamma}(\mathbf{x}, t)=\tilde{\gamma}(\mathbf{x}, t) h_{x}^{t}, h_{y}^{t}$ and with core radius $\sigma=\max \left(h_{x}^{t}, h_{y}^{t}\right)$. On the other hand, a vortex element is eliminated if it leaves the domain or jumps into the thin tile region. 


\subsection{Exit boundary condition}

The most commonly applied boundary condition in vortex methods is the specification of a uniform potential flux at the exit such that potential continuity is satisfied on the boundary [11]:

$$
\left(\mathbf{u}_{P}\left(\mathbf{x}_{o}\right) \cdot \mathbf{n}\right)_{\text {exit }}=\left(\frac{-1}{S_{\text {exit }}}\right) \int_{\partial D \backslash \partial D_{\text {exit }}} \mathbf{u}_{P}\left(\mathbf{x}_{o}\right) \cdot \mathbf{n} d S\left(\mathbf{x}_{0}\right), \quad \mathbf{x}_{o} \in \partial D
$$

This formulation yields an incorrect slip velocity at the walls circumscribing the exit surface. However, extending the channel length by one to two channel heights produces satisfactory results upstream of the exit [11] — at the expense of added computational cost.

We introduce the following two dimensional Poisson equation at the exit surface which enforces a fully developed velocity profile there; i.e., $(\partial \mathbf{u} \cdot \mathbf{n} / \partial n)_{\text {exit }}=0$ :

$$
\begin{aligned}
\nabla^{2} \Phi\left(\mathbf{x}_{o}\right)=-\frac{\partial \mathbf{u}_{\omega}\left(\mathbf{x}_{o}\right) \cdot \mathbf{n}}{\partial n} & \mathbf{x}_{o} \in \partial D \\
\nabla \Phi\left(\mathbf{x}_{o}^{\prime}\right) \cdot \hat{\mathbf{n}}=-\mathbf{u}_{P}\left(\mathbf{x}_{o}^{\prime}\right) \cdot \hat{\mathbf{n}}=-\left(\mathbf{u}\left(\mathbf{x}_{o}^{\prime}\right)-\mathbf{u}_{\omega}\left(\mathbf{x}_{o}^{\prime}\right)\right) \cdot \hat{\mathbf{n}} & \mathbf{x}_{o}^{\prime} \in \partial(\partial D)
\end{aligned}
$$

where the subscript "exit" is eliminated from the equations for the sake of simplicity, $\partial D$ is the exit plane and $\partial(\partial D)$ is its boundary. $\hat{\mathbf{n}}$ is the unit outward normal to the boundary of the exit plane, and in the exit plane. The Poisson equation is solved by the finite element method using the grid distribution for the boundary elements. Given the potential distribution at the exit, the three-dimensional Laplace equation that is used to impose the potential flow field admits a mixed Dirichlet-Neumann boundary condition and has a unique solution.

\section{Results}

\subsection{Flow in a duct}

In this section, we present results from a parametric study of the flow in a duct with square crosssection. We chose this simple geometry because of the availability of an exact solution for the fully developed velocity profile [20]. The purpose of these simulations is to examine the accuracy of the method by comparing the numerical results with the analytical solution and to evaluate the convergence properties as the numerical parameters are varied.

Six cases are examined in this paper. The Reynolds number based on the duct height $H$ and uniform inlet velocity $U_{\text {in }}$ is set at 100 . The ratio of the duct length $L_{c}$ to its height is set at 7 , which satisfies the condition for a fully developed velocity profile at the exit: $\frac{L_{c}}{H} \geq \frac{R e}{20}[21]$. The relevant parameters in this study are listed in Table 1. The tile and (quadratic) boundary element resolutions in Table 1 refer to the number of rectangular elements per duct wall. The origin is set at a corner of the duct inlet, with the $z$ coordinate pointing toward the exit surface. In all cases, the no-slip boundary condition is applied at the walls and the inlet velocity is fixed at $U_{\text {in }}=1.0$. The exit boundary condition is satisfied by either the standard or the fully developed exit velocity formulation - as shown in the table. The flow is initialized using a potential field with a uniform velocity, $U_{\text {in }}=1.0$, throughout the duct. This sets the slip velocities and generates vorticity at the channel walls.

In all cases the number of vortex elements reaches a plateau at $t<10$, signifying the end of the transient between the flow startup and the onset of stationary state. The flow field is obtained by averaging the instantaneous solutions over several timesteps to eliminate the statistical errors associated with the random walk simulation of diffusion. For the following comparison, the results 
Table 1

\begin{tabular}{cccccccc}
\hline \hline Case & BLRP & BLTC & $\Delta t$ & $\begin{array}{c}\text { Time Step } \\
\text { Resolution }\end{array}$ & $\begin{array}{c}\text { REM } \\
\text { Resolution }\end{array}$ & $\begin{array}{c}\text { Tile } \\
\text { Condition }\end{array}$ & Boundary \\
\hline $\mathbf{1}$ & 4 & 1.5 & 0.1 & 300 & $4 \times 24$ & $3 \times 21$ & Std. \\
$\mathbf{2}$ & 2 & 1.5 & 0.1 & 300 & $4 \times 24$ & $3 \times 21$ & Std. \\
$\mathbf{3}$ & 8 & 1.5 & 0.1 & 300 & $4 \times 24$ & $3 \times 21$ & Std. \\
$\mathbf{4}$ & 4 & 1.0 & 0.1 & 300 & $4 \times 24$ & $3 \times 21$ & Std. \\
$\mathbf{5}$ & 4 & 0.5 & 0.1 & 600 & $4 \times 24$ & $3 \times 21$ & Std. \\
$\mathbf{6}$ & 4 & 0.5 & 0.1 & 600 & $4 \times 24$ & $3 \times 21$ & Ful. Dev. \\
\hline \hline
\end{tabular}

from times 20.0 to 30.0 were sampled at 5 timestep intervals and averaged over a total of 20 samples. The exception to this was Case 5 where a total of 60 samples were averaged from 20.0 to 50.0.

Figure 1 depicts the streamwise velocity profile as a function of BLRP for Cases 1 through 3 at four locations on the $z=4.5$ surface. Recall that increasing the value of BLRP is analogous to concentrating more grid points in the vicinity of the walls and is thus expected to improve the field resolution there. However, increasing BLRP from 2 to 8 does not improve the velocity field within the domain appreciably and, as the figure shows, the profiles are almost indistinguishable at $z=4.5$.

Cases 1, 4 and 5 represent a convergence study as a function of BLTC. Figure 2 depicts a comparison between the streamwise velocity profiles for the three cases and the exact solution at four locations on the $z=4.5$ surface. It is clear from the figure that BLTC influences the convergence significantly. In essence, the reduction in BLTC is equivalent to the decrease in the thickness of the boundary region, which in turn improves the validity of the assumptions behind the tile approximation and equations (6).

Case 6 is devised to test the effect of the proposed fully developed exit boundary condition versus the standard boundary condition applied to Case 5. A survey of the velocity profiles at selected streamwise stations verifies the similarity of the flow field up to $z=5.0$, beyond which the two solutions diverge from each other. Figure 3 shows the effect of the exit boundary condition on the exit velocity profile obtained in Cases 5 and 6 . The relative accuracy of the fully developed exit boundary condition is evident in the figure. Consequently, the benefit of applying our proposed exit condition is a 30 percent reduction in the computational domain for this particular geometry and a correspondingly reduced computational cost.

\subsection{Piston driven flow in a cylinder}

In this section, we present results from the simulation of piston driven flow inside a cylinder equipped with a diagonally off-centered intake port (with square cross-sections.) All dimensions are normalized by the cylinder width $D_{c}$, and the velocity is normalized by the maximum piston speed at mid-stroke, $V_{m}$. The Reynolds number based on $D_{c}$ and $V_{m}$ is set at 350 . The port dimensions are 0.4 in all directions. The port faces are parallel to the cylinder faces and its axis is diagonally off-centered from the cylinder axis by $(0.15 \times 0.15)$. The cylinder volume is 0.15 when the piston is at rest at its top location, TDC, and it is 1.0 at the bottom location, BDC. No-slip boundary condition is applied to all solid boundaries. The flow is generated by moving the piston from rest at TDC according to a cosine function for the displacement and a sine function for the piston speed. The uniform flow through the inlet of the port is obtained by assigning no-flux boundary condition to the remaining boundary surfaces and satisfying the continuity requirement. The induction process is discretized using 200 equal timesteps. Other parameters of importance are as follows: 
$\mathrm{BLRP}=2, \mathrm{BLTC}=1.5$.

A time varying number of boundary elements (and tiles) is used to discretize the cylinder walls, which undergo a length change due to the piston motion. The criterion is to assign a maximum number of linear boundary elements (and tiles) $N_{\max }$ to discretize the cylinder walls when the piston is at $\mathrm{BDC}-L_{\max }$. The instantaneous number of elements $N$ is obtained using the relation $N=\max \left(1, N_{\max } L_{c} / L_{\max }+0.5\right)$, where $L_{c}$ is the instantaneous length of the cylinder wall. In addition to saving CPU time, this approach maintains a nominal elemental mesh size in the order of $L_{\max } / N_{\text {max }}$. In contrast, selecting a fixed value for $N$ would yield extremely thin elements at the beginning of the run, and would generate inaccurate solutions. Each of the four plates comprising the intake port is meshed using $8 \times 8$ linear boundary elements and $4 \times 4$ tiles. The expansion face is discretized using $16 \times 16$ boundary elements and $10 \times 10$ tiles. The maximum number of boundary elements and tiles are set at 16 and 8, respectively, along the cylinder walls. A cosine function is used to cluster the boundary elements near the sharp edges and corners; however, the tiles are distributed uniformly.

Figure 4 depicts the side view of the instantaneous position and velocity of the vortex elements within a slice of volume with thickness 0.15 and plane of symmetry which passes through the plane of symmetry of the inlet port. The circles represent the element positions and not the vector heads. The color coding is based on the instantaneous maximum velocity in the field. Notice that as the piston accelerates from TDC to its peak speed at $90^{\circ}$ crank angle the boundary layers on the intake port walls experience a corresponding decrease in their thickness. This is to be expected, because the harmonic motion of the piston changes the effective Reynolds number in the port as a function of the crank angle. The peak Reynolds number based on the port parameters is 875 .

During the induction stage a square jet is issued from the intake port into the chamber. The jet separates from the port due to the sudden expansion at the interface of the port and the chamber and begins to roll up in the cylinder into a square-shaped torus. The cross-section of the torus is depicted in the figure as two distinct eddies on the two sides of the jet. The high-speed vortex elements in the cylinder, which extend from the intake port boundary layer elements, represent the shear layer caused by the momentum difference between the high-speed jet and the relatively slow vortical field in the chamber. In the early stages of the flow, up to around $60^{\circ}$ crank angle, the curvature and the orientation of the left and right shear layers are determined by the piston position (crank angle) and the relative position of the port with respect to the cylinder. This can be verified by recognizing that each shear layer is streamlined such that a curve through it extrapolates out to the intersection of the cylinder wall and the piston. As the piston moves down, the eddies entrain most of the fluid from the jet and some of the fluid which already exists in the cylinder. By $54^{\circ}$ crank angle, the left eddy fully occupies the area under the left expansion face, whereas the right eddy develops to its fullest at approximately $72^{\circ}$ crank angle. As a result of the jet entrainment and volumetric expansion of the cylinder due to the piston motion, both eddies continue to grow along the axial direction and expand in the radial direction. The left eddy bulges beyond the width of the left expansion face and tilts the jet further to the right.

Figure 5 depicts the perspective view of the trajectory of the vortex elements generated by the piston face due to its interaction with the intake jet. The fixed length vectors represent vorticity and the colors represent their magnitude normalized by the maximum volumetric vorticity in the field within the duration of the simulation. The jet at the throat of the intake port is 6.25 times faster than the moving piston. Consequently, it impinges upon the piston almost instantly and disperses out radially, generating semicircular vortex rings which are parallel to the piston face. The direction of these rings is opposite the direction of the toroidal vortex in the cylinder. The figure shows that an initially coherent ring of vorticity develops azimuthal instability and streamwise vorticity, due to its interaction with the chamber walls and the jet-induced vortex torus. 


\section{Conclusion}

A grid-free numerical scheme, based on the random vortex and boundary element methods, is outlined for the simulation of time-dependent, incompressible flow in three-dimensional domains. The computational domain is decomposed into a thin near-boundary region and an interior domain. The evolution of vorticity within the interior is approximated by the random vortex method using spherical vortex elements. The vorticity field near the boundary is discretized using thin rectangular vortex tiles and the flow is evaluated by a Prandtl-type simplification of the Navier-Stokes equations. A direct boundary element method is used to evaluate the potential flow that satisfies the noflux boundary condition. An extended tile generation mechanism is developed to impose the noslip boundary condition on fixed as well as time-varying walls. Additionally, an exit boundary condition is proposed which enforces zero streamwise velocity gradient at the exit. In this paper, the convergence of the method, its accuracy, and the effect of the exit boundary condition on the solution are demonstrated (for a limited set of parameters) using the case of flow in a duct with square cross-section at $R e=100$. Good agreement is obtained with the exact solution with relatively low resolution. Preliminary results suggest that, for the range of parameters tested, the solution is relatively insensitive to BLRP (the tile resolution in the direction normal to the wall), and is a strong function of BLTC (the thickness of the near-boundary region where the Prandtl-type approximation is applied). Furthermore, the proposed exit boundary condition was shown to be superior to that used in the past. This allowed us to reduce the length of the computational domain by two duct widths, and hence reduce the CPU time substantially. The method is also applied to the more complex case of flow inside a square cylinder with a harmonically moving piston at $R e=350$ based on the piston width and its speed at mid-stroke. The results show that the square jet emanating from the inlet port expands inside the chamber, and quickly develops into a coherent square shaped vortical torus in the cylinder. In addition, the interaction of the jet with the piston face generates a vortex ring parallel to the piston and counter-rotational with respect to the square torus.

\section{Acknowledgments}

This project was funded by FORD Motor Company. The numerical experiments were performed on the Cray YMP at the National Center for Supercomputing Applications, and on the Cray C90 at the Pittsburgh Supercomputing Center.

\section{References}

[1] Anderson, C.R. and Greengard, C., "On Vortex Methods," SIAM J. Num. Anal., 22 (3), pp. 413, 1985.

[2] Beale, J. and Majda, A., "Vortex Methods for Fluid Flow in Two or Three Dimensions," in Contemporary Mathematics, Amer. Math. Soc., 28, pp. 221, 1984.

[3] Beale, J. and Majda, A., "Rates of Convergence for Viscous Splitting of the Navier-Stokes Equations," Math. Comp., 37 (156), pp. 243, 1981.

[4] Beale, J. and Majda, A., "Vortex Methods I: Convergence in Three Dimensions," Math. Comp., 39 (159), pp. 1, 1982. 
[5] Beale, J. and Majda, A., "Vortex Methods II: Higher Order Accuracy in Two and Three Dimensions," Math. Comp., 39 (159), pp. 29, 1982.

[6] Chorin, A.J., "Vortex Sheet Approximation of Boundary Layers," J. Comp. Phys., 27, pp. 428, 1978.

[7] Chorin, A.J., "Vortex Models and Boundary Layer Instability," SIAM J. Sci. Stat. Comp., 1 (1), pp. 1, 1980.

[8] Fishelov, D., "Vortex Methods for Slightly Viscous Three-Dimensional Flow," SIAM J. Sci. Stat. Comp., 11 (3), pp. 399, 1990.

[9] Gharakhani, A., "3-D Vortex-Boundary Element Method for the Simulation of Unsteady, High Reynolds Number Flows," Sc.D. Thesis, Dept. of Mech. Eng'n., M.I.T., Cambridge, MA, 1995.

[10] Gharakhani, A. and Ghoniem, A.F., "BEM Solution of the 3D Internal Neumann Problem and a Regularized Formulation for the Potential Velocity Gradients," to be published in Int. J. Num. Meth. Fluids.

[11] Ghoniem, A.F. and Ng, K., "Numerical Study of the Dynamics of a Forced Shear Layer," Phys. Fluids, 30 (3), pp. 706, 1987.

[12] Ghoniem, A.F. and Gagnon, Y., "Vortex Simulation of Laminar Recirculating Flow," J. Comp. Phys., 68, pp. 346, 1987.

[13] Goodman, J., "Convergence of the Random Vortex Method," Comm. Pure Appl. Math., 40 (2), pp. 189, 1987.

[14] Hald, O., "Convergence of Vortex Methods For Euler's Equations, II," SIAM J. Num. Anal., 16 (5), pp. 726, 1979.

[15] Knio, O.M. and Ghoniem, A.F., "Three-Dimensional Vortex Simulation of Rollup and Entrainment In A Shear Layer," J. Comp. Phys., 97 (1), pp. 172, 1991.

[16] Leonard, A., "Vortex Methods for Flow Simulation," Ann. Rev. Fluid Mech., 17, pp. 523, 1985.

[17] Long, D.-G., "Convergence of the Random Vortex Method In Two Dimensions," J. Amer. Math. Soc., 1 (4), pp. 779, 1988.

[18] Martins, L.-F. and Ghoniem, A.F., "Vortex Simulation of the Intake Flow In A Planar PistonChamber Device," Int. J. Num. Meth. Fluids, 12, pp. 237, 1991.

[19] Puckett, E.G., "A Study of the Vortex Sheet Method and Its Rate of Convergence," SIAM J. Sci. Stat. Comp., 10 (2), pp. 298, 1989.

[20] Rouse, H., Adv. Mech. Fluids, John Wiley \& Sons, Inc., New York/London/Sydney, 1965.

[21] Schlichting, H., Boundary Layer Theory, McGraw Hill Book Co., 1968.

[22] Sladek, V. and Sladek, J., "Non-singular Boundary Integral Representation of Potential Field Gradients," Int. J. Num. Meth. Eng'n., 33, pp. 1181, 1992.

[23] Sladek, V. and Sladek, J., "Non-singular Boundary Integral Representation of Stresses," Int. J. Num. Meth. Eng'n., 33, pp. 1481, 1992. 

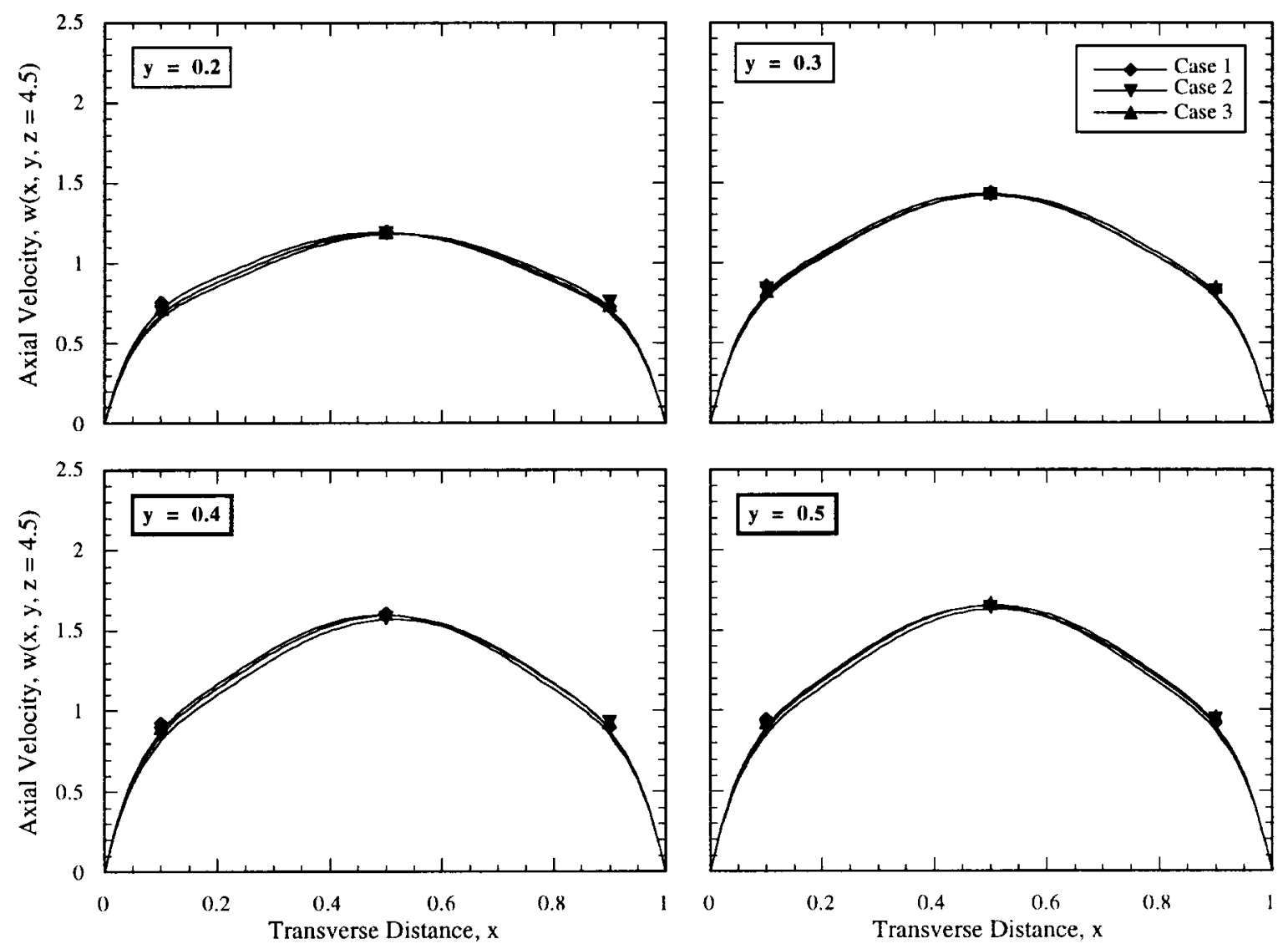

Figure 1: Effect of BLRP on the streamwise velocity at $z=4.5$.

[24] Winckelmans, G.S. and Leonard, A., "Contributions to Vortex Particle Methods for the Computation of Three-Dimensional Incompressible Unsteady Flows," J. Comp. Phys., 109, pp. 247, 1993. 

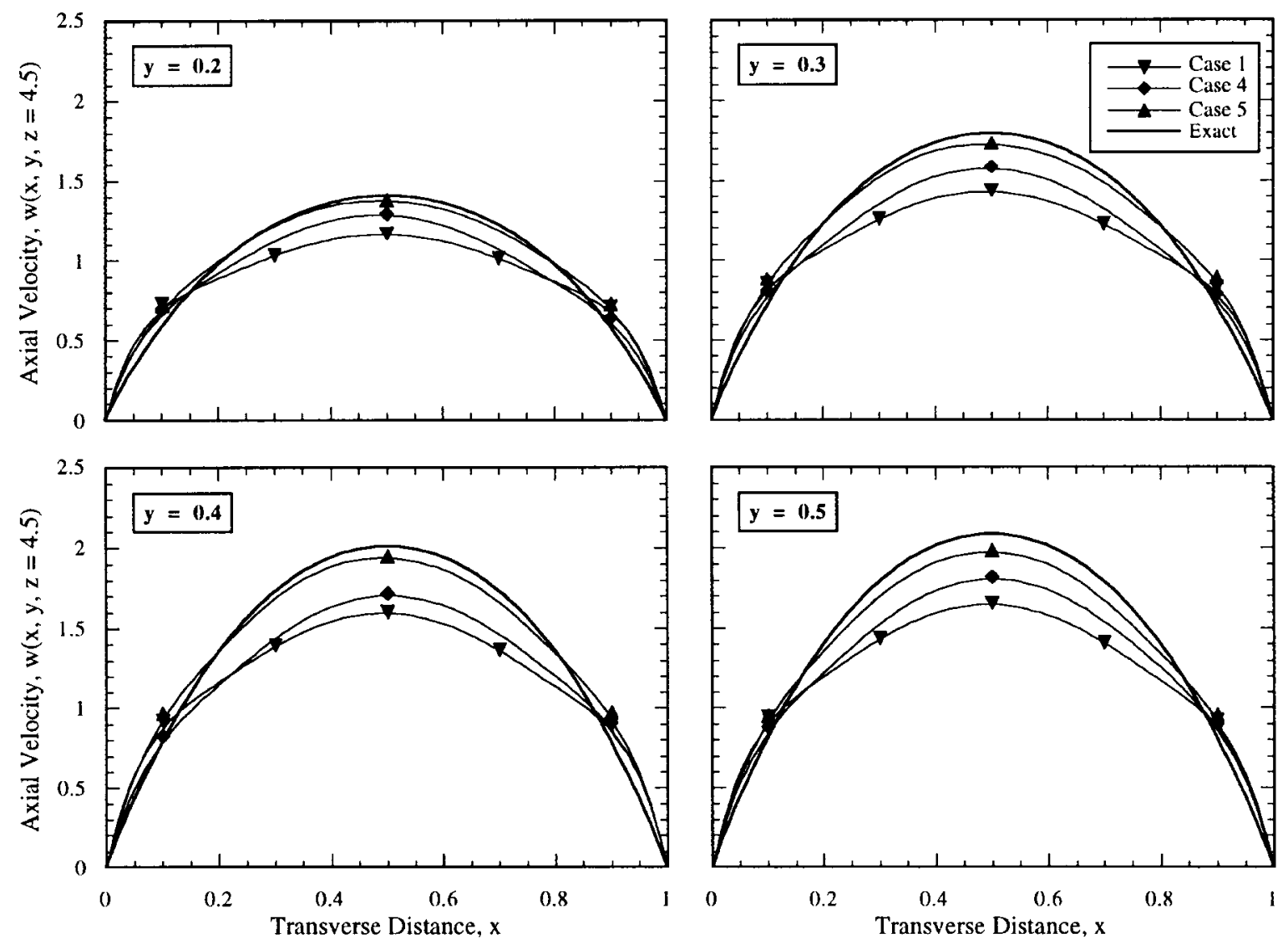

Figure 2: Effect of BLTC on the streamwise velocity at $z=4.5$. 

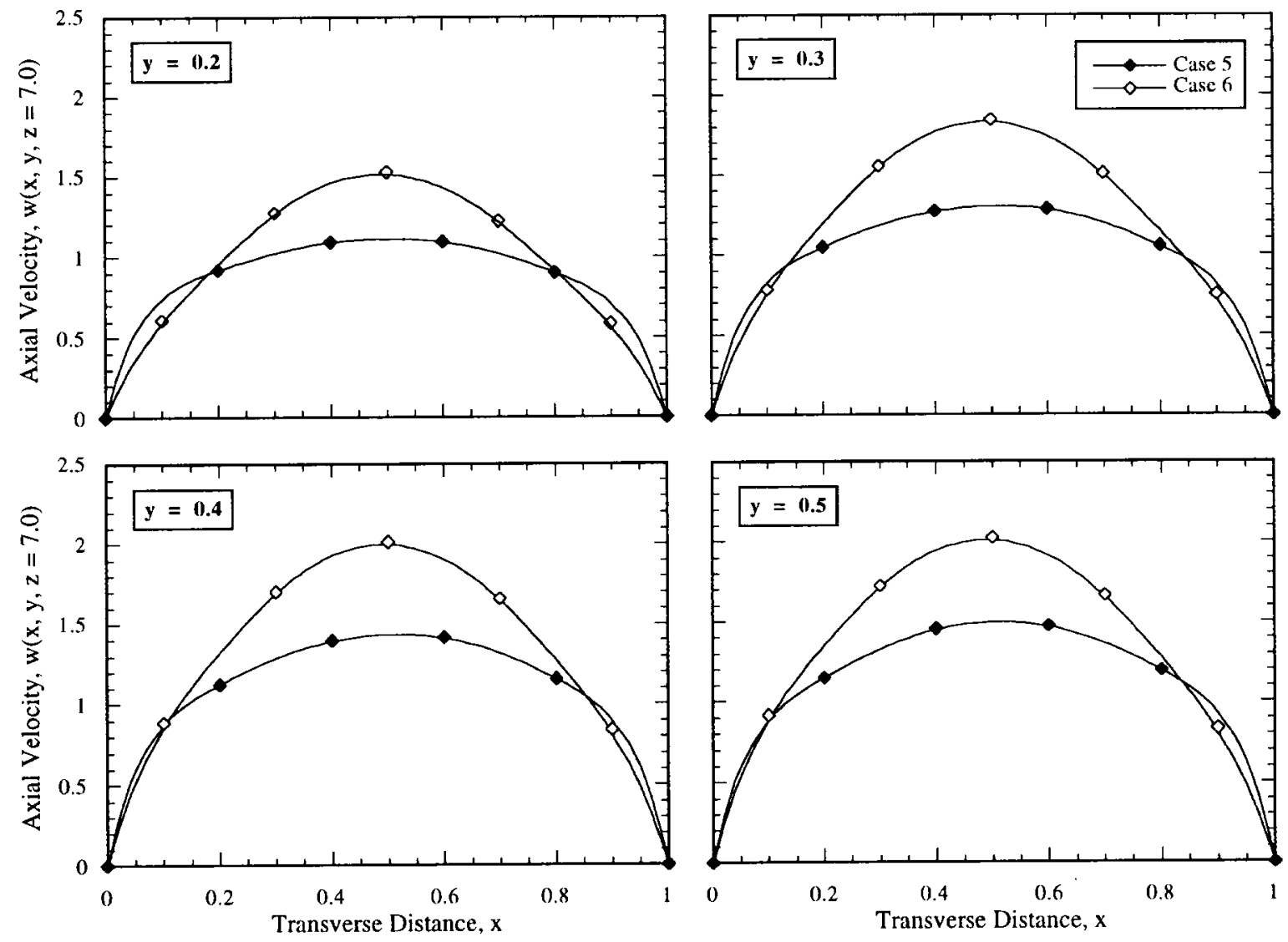

Figure 3: Effect of the exit boundary condition on the velocity profile at the exit surface. 


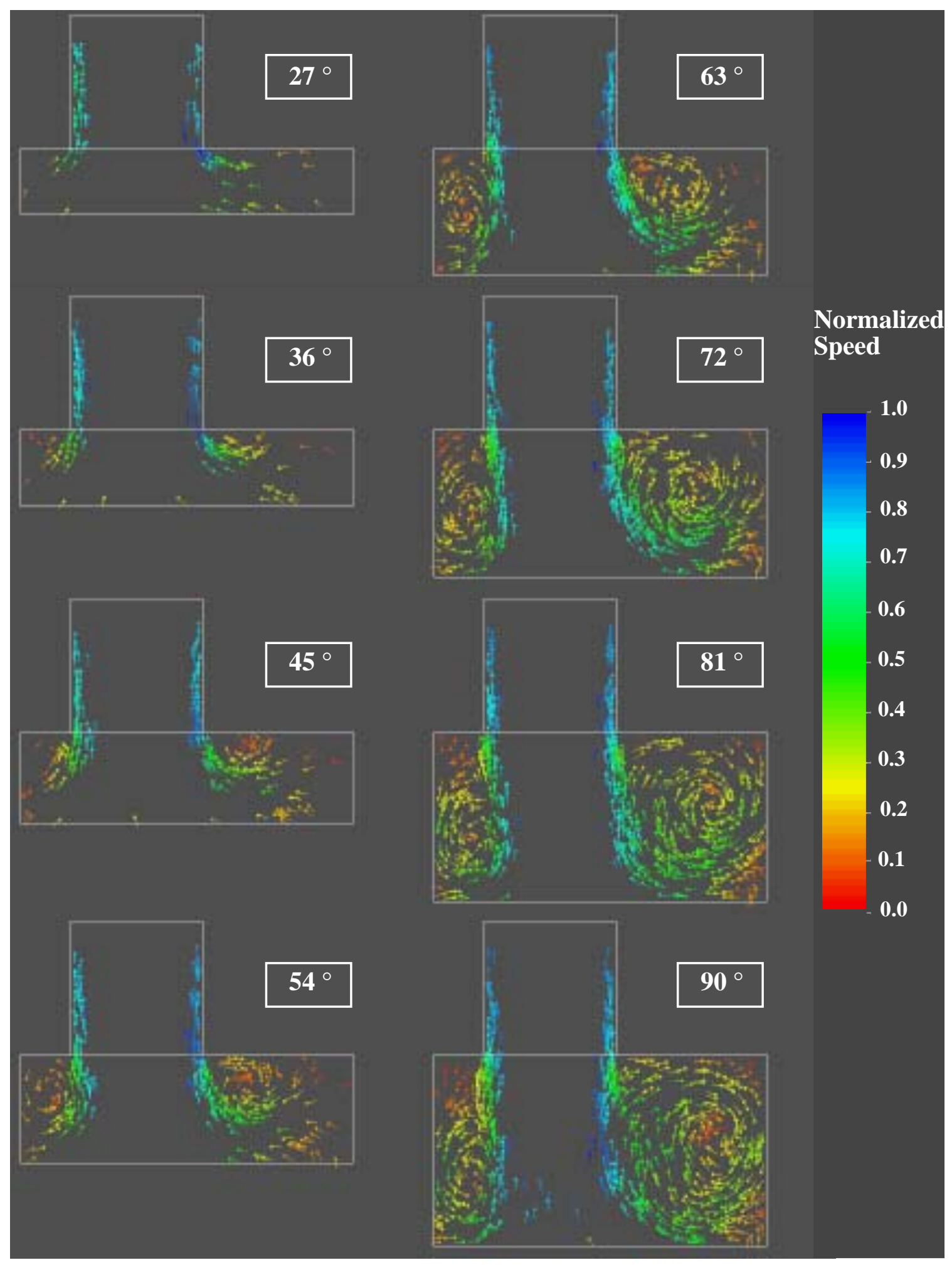

Figure 4: Vortex element trajectories within a volume of thickness 0.15 , centered at the symmetry plane of the port. Normalization in each time frame is based on the instantaneous maximum speed in the field. Velocity vectors are fixed in length. 


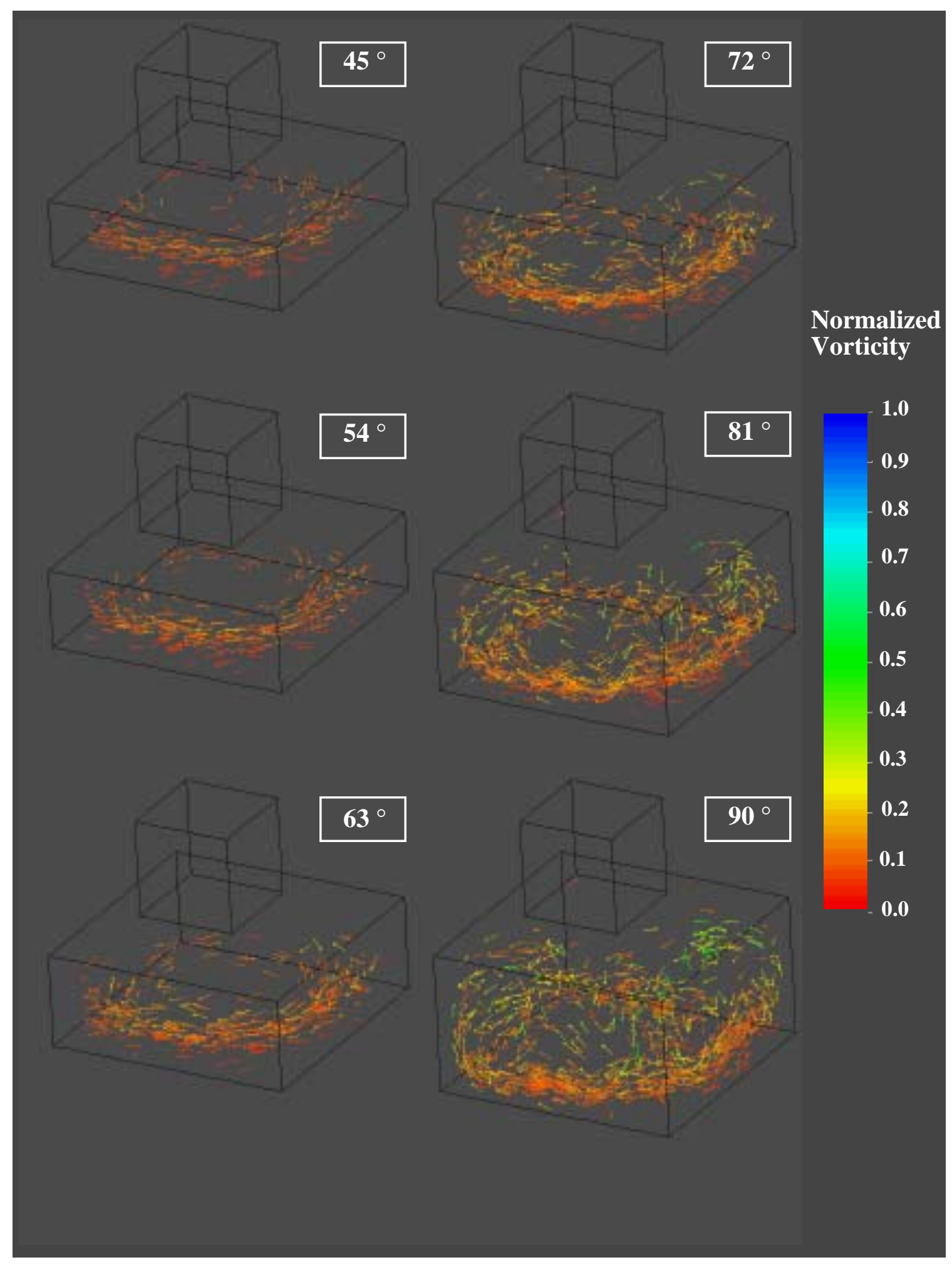

Figure 5: Perspective view of the vortex element trajectories generated at the piston face. Vorticity vectors are fixed in length. Normalization is based on the maximum volumetric velocity in the field and for the duration of the induction step. 